\title{
Study of tau-pair production at HERA
}

\author{
Masaki Ishitsuka* \\ Tokyo Institute of Technology \\ E-mail: ishitsukadphys.titech.ac.ip
}

for ZEUS Collaboration

\begin{abstract}
A study of tau-pair production has been performed with the ZEUS detector at HERA ep collider, using a data sample corresponding to an integrated luminosity of $0.33 \mathrm{fb}^{-1}$. The tau candidates were identified from their decays into electrons, muons or hadronic jets. The observed tau-pair candidates have been compared with the prediction from the Standard Model, in which BetheHeitler processes are expected to be dominant. The total cross section was extracted from the number of tau-pair candidates, to be consistent with the prediction from the Standard Model. The measured final-state distributions agreed well with the prediction, and no significant excess was found at high invariant mass of the tau pair.
\end{abstract}

36th International Conference on High Energy Physics,

July 4-11, 2012

Melbourne, Australia

${ }^{*}$ Speaker. 


\section{Introduction}

Production of isolated-lepton pairs at ep collision is dominated by Bethe-Heitler process, $\gamma \gamma \rightarrow l^{+} l^{-}$, and accurately predicted by electroweak interaction in the Standard Model (SM) [四]. Therefore, measurement of the lepton-pair production at the $e p$ collider HERA provides a test of the SM, and deviations of the event yield or final-state distributions from the prediction, if that is observed, can be interpreted as a hint of the new physics beyond the SM. ZEUS and H1 Collaborations have published a study of multi-electron and multi-muon events based on the entire statistics collected at HERA I and II, in which no significant deviation from the SM has been observed [వ]. Further investigations of multi-lepton production were performed in tau-pair production by $\mathrm{H} 1$ based on HERA I data [3].

In this paper, we report on a study of tau-pair production with the ZEUS detector, using a data sample collected in HERA II running phase [ [ 7 ]. The tau candidates are identified from their decays into electrons, muons or hadronic jets.

\section{Experimental set-up}

The data for this analysis were collected between 2004 and 2007 at HERA ep collider, referred to as HERA II running period, using the ZEUS detector with an integrated luminosity of $334 \mathrm{pb}^{-1}$. During this period, HERA operated with an electron or positron beam with an energy of $27.5 \mathrm{GeV}$ and a proton beam with $920 \mathrm{GeV}$. Among the total integrated luminosity, $179 \mathrm{pb}^{-1}$ data were taken with $e^{-} p$ collision, while the rest $155 \mathrm{pb}^{-1}$ were taken with $e^{+} p$ collision. The lepton beams were polarized with roughly equal periods for right-handed and left-handed polarization, such that the average polarization was negligible.

The ZEUS detector is a multi-purpose detector built at a HERA ep interaction point. Charged particles were tracked in the central tracking detector (CTD), which operated in a magnetic field of $1.43 \mathrm{~T}$ provided by a thin superconducting solenoid. The CTD was complemented by a silicon micro-vertex detector (MVD). The high-resolution uranium-scintillator calorimeter (CAL) consisted of three parts: the forward (FCAL), the barrel (BCAL) and the rear (RCAL) calorimeters. Each part was subdivided longitudinally into one electromagnetic section (EMC) and either one (in RCAL) or two (in BCAL and FCAL) hadronic sections (HAC). The muon system consisted of barrel, rear (B/RMUON) and forward (FMUON) tracking detectors. More detailed description of the ZEUS detector can be found elsewhere [[]].

\section{Event selection}

Signal in this analysis consists of events in presence of two isolated taus with high transverse energy, $e p \rightarrow(e) \tau^{+} \tau^{-} X$, where $X$ represents either proton (elastic reaction), resonant (quasielastic) or hadronic system (inelastic scattering). The scattered electron can be observed in the calorimeter only for interactions with high $Q^{2}$, whose contribution is estimated to be $1 \%$ in ditau candidates.

Events which satisfy the following criteria were first selected at preselection level: (1) $2 \leq$ $N_{t r k} \leq 7$, where $N_{t r k}$ represents the number of tracks in the event; (2) $\left|Z_{V T X}\right|<40 \mathrm{~cm}$, where $Z_{V T X}$ 
is the $Z$ coordinate of the interaction vertex reconstructed from the tracks; (3) $E_{F C A L}^{I R}<1 \mathrm{GeV}$, where $E_{F C A L}^{I R}$ is the sum of the energy deposits in the CAL cells in the first inner ring around the forward beam-pipe; (4) $E-P_{Z}<60 \mathrm{GeV}$, where $E$ and $P_{Z}$ are the sum of the total and longitudinal energy deposits in the calorimeter and muon track. (1) was required from the topology of ditau production process. (3) was applied in order to suppress the dominant background from deep inelastic scattering (DIS) neutral current (NC), ep $\rightarrow e X$, and photoproduction, $\gamma p \rightarrow X$. The major signal processes were therefore elastic and quasi-elastic reactions where the final-state proton escapes in the forward beam-pipe. Contributions from elastic and quasi-elastic reactions were estimated from the MC simulation to be $71 \%$ and $27 \%$, respectively.

The two tau leptons were identified from their decays into an electron, a muon or hadrons. The topologies in which the two tau leptons both decay into electrons or muons were not considered due to irreducible background of dielectron and dimuon processes.

\subsection{Identification of electrons and muons}

An algorithm which combined information from the energy deposits in the calorimeter with tracks was used to identify electron candidates. The electron candidates were required to have a transverse energy $E_{T}^{e}>2 \mathrm{GeV}$, to be in the polar-angle range $17^{\circ}<\theta_{e}<160^{\circ}$ and to have a good track matched to the calorimeter deposit. The muon candidates were reconstructed in the rear or barrel muon chambers and required to be matched to a good track and to a calorimeter energy deposit. The muon candidates were required to have a transverse momentum $p_{T}^{\mu}>2 \mathrm{GeV}$ and to be in the polar-angle range $34^{\circ}<\theta_{\mu}<157^{\circ}$. Isolation conditions were further applied to both electron and muon candidates.

\subsection{Identification of hadronic tau decays}

The jets deriving from decay of tau leptons were reconstructed from the CAL cells using the $k_{T}$ cluster algorithm, assuming massless objects. The jets were required to have a transverse energy $E_{T}^{j e t}>5 \mathrm{GeV}$ and pseudorapidity $\left|\eta^{j e t}\right|<2$. Following two conditions were further applied to reject electrons faking hadronic tau decays: at least one good track associated with the jet should exist in a $\eta-\phi$ cone of radius 1 centered on the jet axis; fraction of jet energy in the electromagnetic section of the calorimeter should satisfy $f_{E M C}<\min \left(0.95,2 \cdot R_{90 \%}+0.7\right)$, where $R_{90 \%}$ is the radius of $\eta-\phi$ cone centered on the jet axis that contains $90 \%$ of the jet energy.

Jet originating from hadronic tau decays are characterized by their low mass, low multiplicity (mostly either one or three tracks) and pencil-like shape in contrast to QCD-induced hadronic jets which typically have broader shape and higher multiplicity. These properties were exploited to discriminate tau jets from QCD jets using multi-variate discrimination technique [目] which was used in the previous publication on single-tau production [ $[\mathbf{}]$. The following six variables were employed: the first and second moment of the radial extension of the jet energy deposition $\left(R_{\text {mean }}\right.$ and $\left.R_{R M S}\right)$; the first moment of the energy deposition in the direction along the jet axis $\left(L_{\text {mean }}\right)$; the number of sub-jets within the jet $\left(N_{s u b j}\right)$; the visible invariant mass of the jet $\left(M_{j e t}\right)$; and the sum of the distance in the $\eta-\phi$ plane between the jet axis and the tracks associated with the jet $\left(R_{t r k}=\sum_{i}^{N_{t r k}} \sqrt{\Delta \eta_{i}^{2}+\Delta \phi_{i}^{2}}\right)$. Distributions of the six variables are shown in Figure $\mathbb{W}$. 


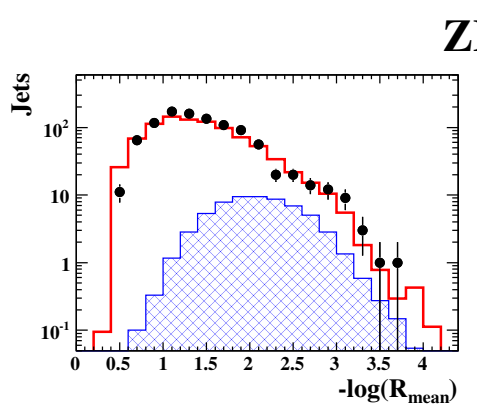

ZEUS
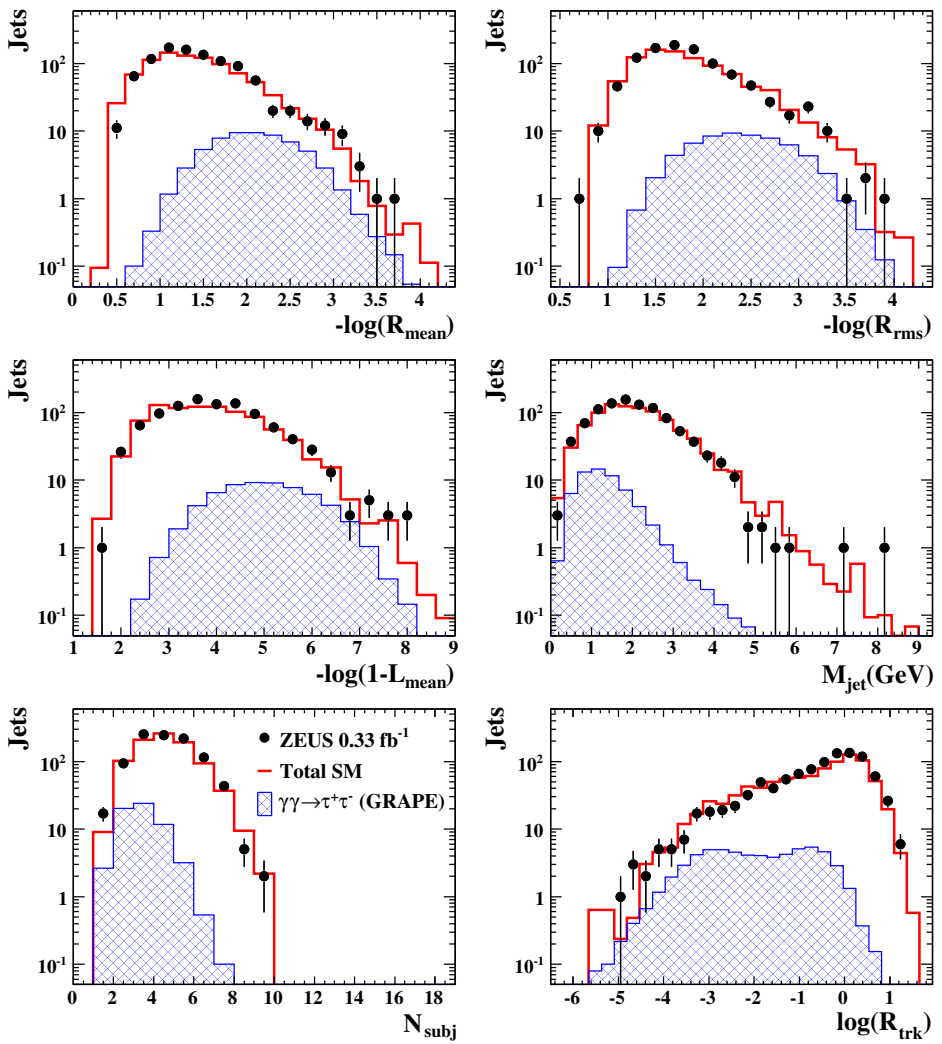

Figure 1: Distributions of the variables used for tau jet discriminant. Points show the data overlaid with the sum of the MC expectation (solid lines) and contribution from ditau signal by GRAPE [区] after all selection criteria except the discriminant cut were applied.

The six variables were combined in a discriminant $D$ given for each point in the phase space $\vec{x}\left(-\log \left(R_{\text {mean }}\right),-\log \left(R_{r m s}\right),-\log \left(1-L_{\text {mean }}\right), N_{\text {subj }}, M_{\text {jet }}, \log \left(R_{\text {trk }}\right)\right)$ by:

$$
D(\vec{x})=\frac{\rho_{\text {sig }}(\vec{x})}{\rho_{\text {sig }}(\vec{x})+\rho_{b k g}(\vec{x})}
$$

where $\rho_{s i g}$ and $\rho_{b k g}$ are the density functions of the signal and the background, respectively. The densities were determined from single-tau MC events for the signal and DIS NC events for the background. Figure $\square$ shows the distributions of the discriminant for each decay topology.

\subsection{Final selection}

After preselection and identification of tau decay candidates, the following additional cuts were applied. In order to further reduce DIS NC background events, tighter containment cut, $E-P_{z}<45 \mathrm{GeV}$, was applied if the scattered electron is not observed. Charge information was used in the following cuts if significance $S_{t r k}=|Q / r| / \sigma(Q / r)$ was greater than 1.5 , where $r$ denotes the radius of the track helix and $\sigma$ is the uncertainty. $Q_{j e t}=\sum_{i} Q_{i, t r k}$ was reconstructed for tau jet candidates if all tracks associated with the jet satisfy $S_{t r k}>1.5$. In the $(e-) e-\mu$ topology, charge 


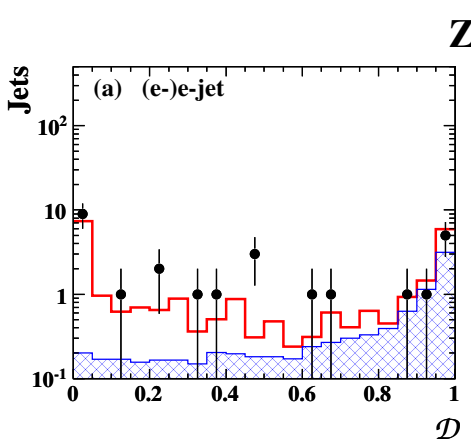

ZEUS
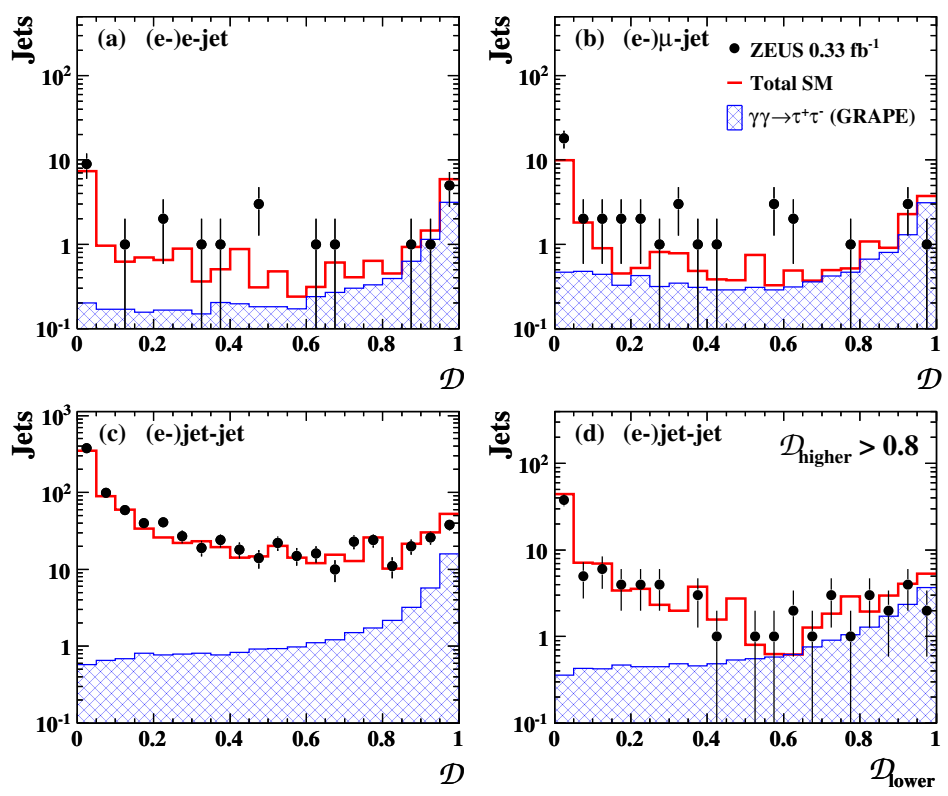

Figure 2: Tau-jet discriminant variable for three decay topologies (a) $(e-) e-j e t$, (b) $(e-) \mu$-jet and (c) $(e-) j e t-$ $j e t$ for events after all selection criteria except the discriminant cut were applied. (d) shows the discriminant for jets with lower $D$ values in $(e-)$ jet-jet channel, after the requirement $D>0.8$ for jets with higher $D$.

of electron and muon $\left(Q_{e}\right.$ and $\left.Q_{\mu}\right)$ were required to be opposite, and $Q_{e}$ to be opposite to that of the beam if $\theta_{e}>1.0$, in order to reduce background due to muon-pair production where one of muons was not observed. In the $(e-) e$-jet topology, $Q_{j e t}$ was required to be equal to \pm 1 and opposite to that of the electron candidate. In the $(e-)$ jet-jet topology, $Q_{j e t}$ were required to be \pm 1 with the two jets having opposite charges. Table $\square$ summarizes the number of tau-pair candidates after all selection criteria ware applied. In total, 25 events were selected in the data while $34.8_{-3.8}^{+3.9}$ events were expected with a purity of $67 \%$ for tau-pair production. A description of the systematic uncertainties can be found in [四]. The total cross section for ditau production was calculated for the kinematic region defined by $p_{T}^{\tau}>5 \mathrm{GeV}$ and $17^{\circ}<\theta^{\tau}<160^{\circ}$, where $p_{T}^{\tau}$ and $\theta^{\tau}$ refer to the transverse momentum and polar angle, respectively, of the tau lepton. The total cross section was found to be

$$
\left.\sigma_{\tau \tau}^{k i n}=3.26 \pm 1.30 \text { (stat. }\right)_{-0.73}^{+0.99} \text { (syst.) pb, }
$$

which is in reasonable agreement with the SM expectation of $5.67 \pm 0.16 \mathrm{pb}$ evaluated from the GRAPE MC generator [ [8]].

Figure 13 shows the visible invariant mass, $M_{\text {visible }}$, and the scalar sum of the visible transverse momenta, $\sum p_{T, \tau \tau}^{v i s i b l e}$, calculated from the two tau candidates. The MC predictions agree well with the data and no excess is observed in the high-mass or high $-\sum p_{T, \tau \tau}^{v i s i b l e}$ region.

\section{Conclusions}

Events with two tau candidates with high transverse momentum have been selected in the 


\begin{tabular}{|c|c|c|c|c||c|}
\hline Topology & $(e-) e-\mu$ & $(e-) e-j e t$ & $(e-) \mu-j e t$ & $(e-) j e t-j e t$ & Total \\
\hline Data & 4 & 7 & 4 & 10 & 25 \\
Total MC & $3.6_{-0.3}^{+1.3}$ & $8.8_{-0.8}^{+1.8}$ & $8.0_{-1.2}^{+2.2}$ & $14.4_{-3.5}^{+2.2}$ & $34.8_{-3.8}^{+3.9}$ \\
$\tau^{+} \tau^{-} \mathrm{MC}$ & $3.0_{-0.2}^{+0.3}$ & $5.3_{-0.2}^{+0.3}$ & $5.9_{-0.5}^{+0.5}$ & $9.0_{-0.3}^{+0.4}$ & $23.2_{-0.7}^{+0.7}$ \\
\hline
\end{tabular}

Table 1: The observed and predicted ditau event yield for each decay topology. The systematic uncertainties are quoted on the MC expectations.

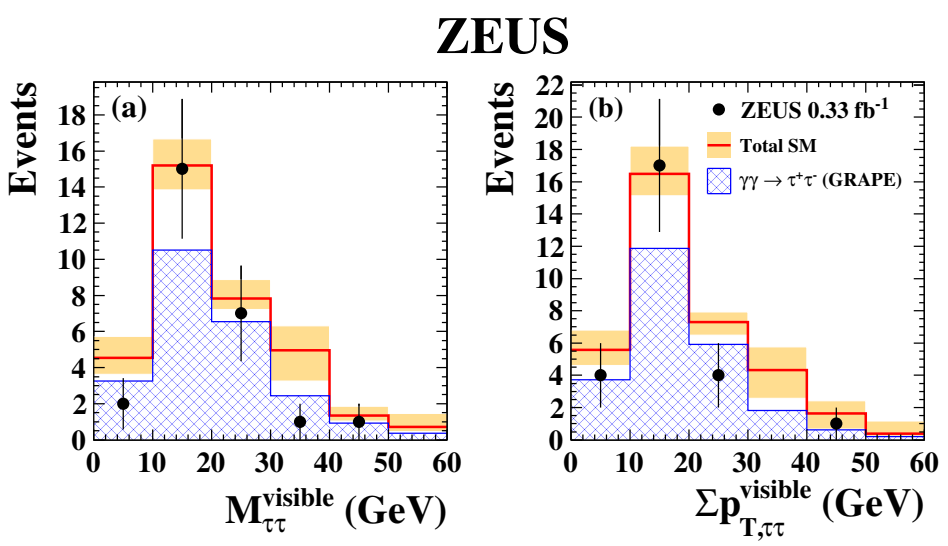

Figure 3: Distriburions of the events after all selection cuts as a function of (a) the visible invariant mass and (2) the scalar sum of the transverse momenta calculated from the two tau candidates.

data collected by the ZEUS experiment and compared with the prediction from the SM. The tau leptons were identified through their decays into electrons, muons or hadronic jets. 25 events were selected in the data while $34_{-3.8}^{+3.9}$ events are expected from the MC simulation including $11.6_{-3.9}^{+3.9}$ of background. Observed distributions of visible invariant mass and transverse momentum are in good agreement with the prediction, and no evidence of physics beyond the Standard Model was found in tau-pair production. The total cross section, in the kinematic region $p_{T}^{\tau}>5 \mathrm{GeV}$ and $17^{\circ}<\theta^{\tau}<160^{\circ}$ was measured to be $\sigma_{\tau \tau}^{k i n}=3.26 \pm 1.30$ (stat. $)_{-0.73}^{+0.99}$ (syst.) pb.

\section{References}

[1] N. Arteaga-Romero, C. Carimalo and P. Kessler, Z. Phys. C 52, 289 (1991).

[2] F.D. Aaron et al. (H1 and ZEUS Collaborations), JHEP 10, 013 (2009).

[3] A. Aktas et al. (H1 Collaboration), Eur. Phys. J. C 48, 699 (2006).

[4] H. Abramowicz et al. (ZEUS Collaboration), JHEP 02, 117 (2011).

[5] ZEUS Collaboration, U. Holm (ed.), The ZEUS Detector. Status Report (unpublished), DESY (1993), available on http://www-zeus.desy.de/bluebook/bluebook.html.

[6] T. Carli and B. Koblitz, Nucl. Inst. Meth. A 501, 576 (2003).

[7] S. Chekanov et al. (ZEUS Collaboration), Phys. Lett. B 583, 41 (2004).

[8] T. Abe, Comp. Phys. Comm. 136, 126 (2001). 